Supporting information

\title{
Latent curing, chemorheological, kinetic, and thermal behaviors of epoxy resin matrix for prepregs
}

Yeong Jae Kim ${ }^{1}$, Sung Ho Choi ${ }^{1}$, Seong Jae Lee* and Keon-Soo Jang*

Department of Polymer Engineering, School of Chemical and Materials Engineering, The University of Suwon, Hwaseong, Gyeonggi 18323, Republic of Korea

1: Y. J. Kim and S.-H. Choi equally contributed; Co- $1^{\text {st }}$ authors

*Corresponding authors: S. J. Lee: sjlee@suwon.ac.kr, K.-S. Jang: ksjang@suwon.ac.kr 

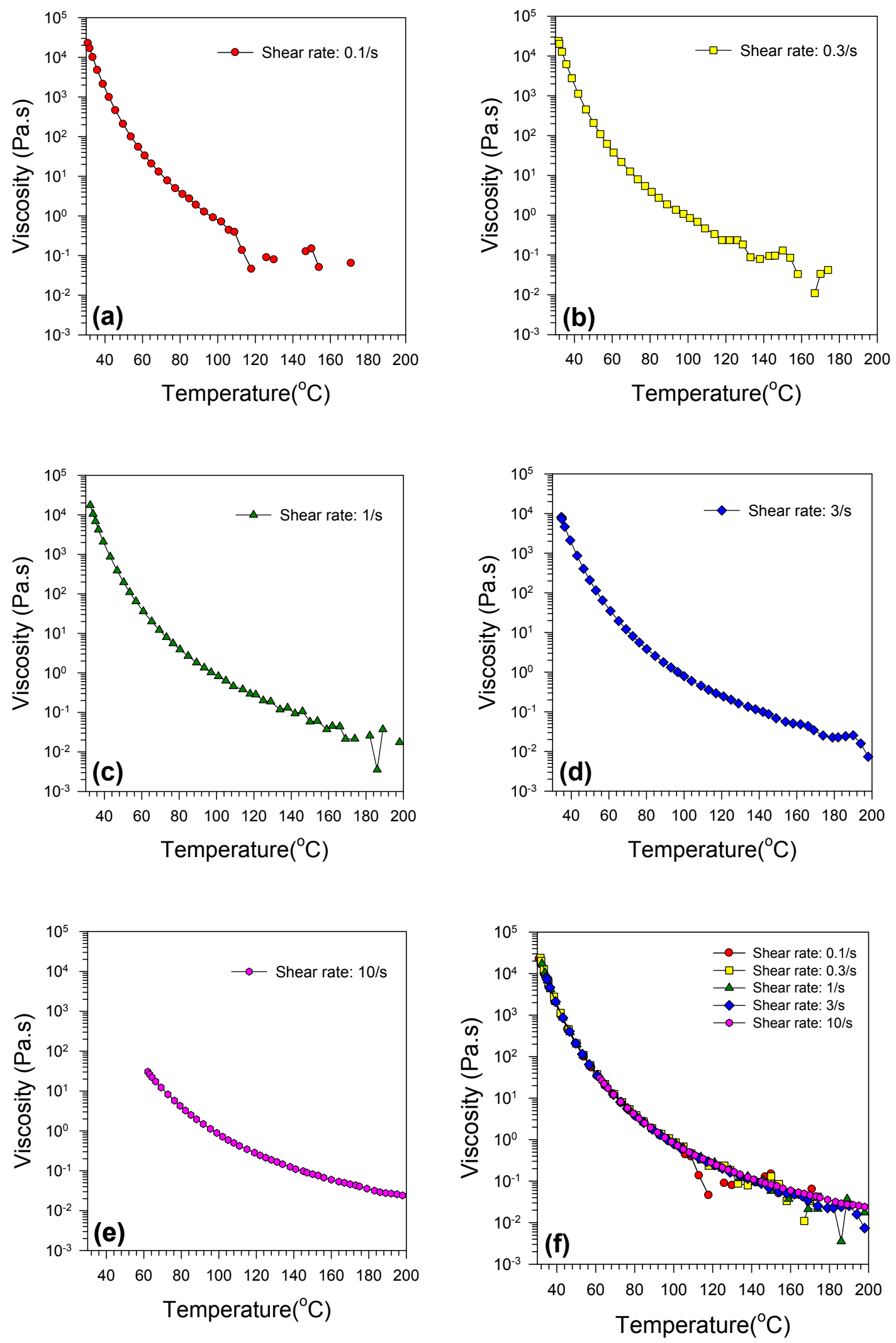

Figure S1. Steady shear viscosity graphs of epoxy mixture (EM) measured at different shear rates (a)-(e), and their superimposed graph (f). The neat epoxy resin mixture showed Newtonian flow behavior from $30^{\circ} \mathrm{C}$ to $200{ }^{\circ} \mathrm{C}$ irrespective of shear rate. 


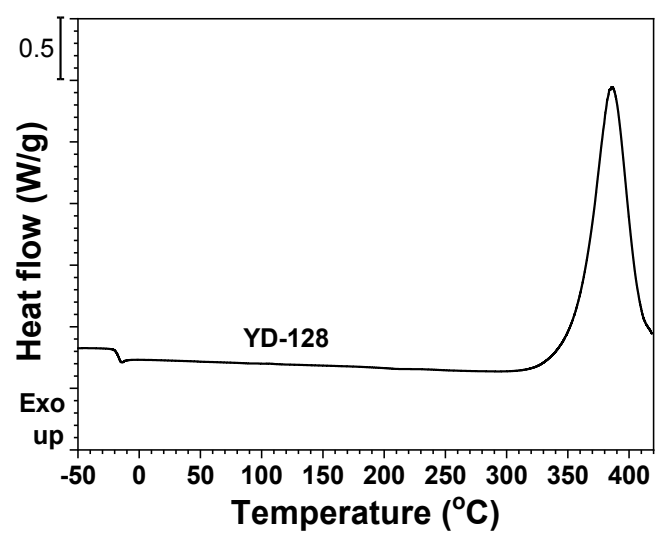

Figure 2. Homopolymerization of epoxy binder measured by DSC. 

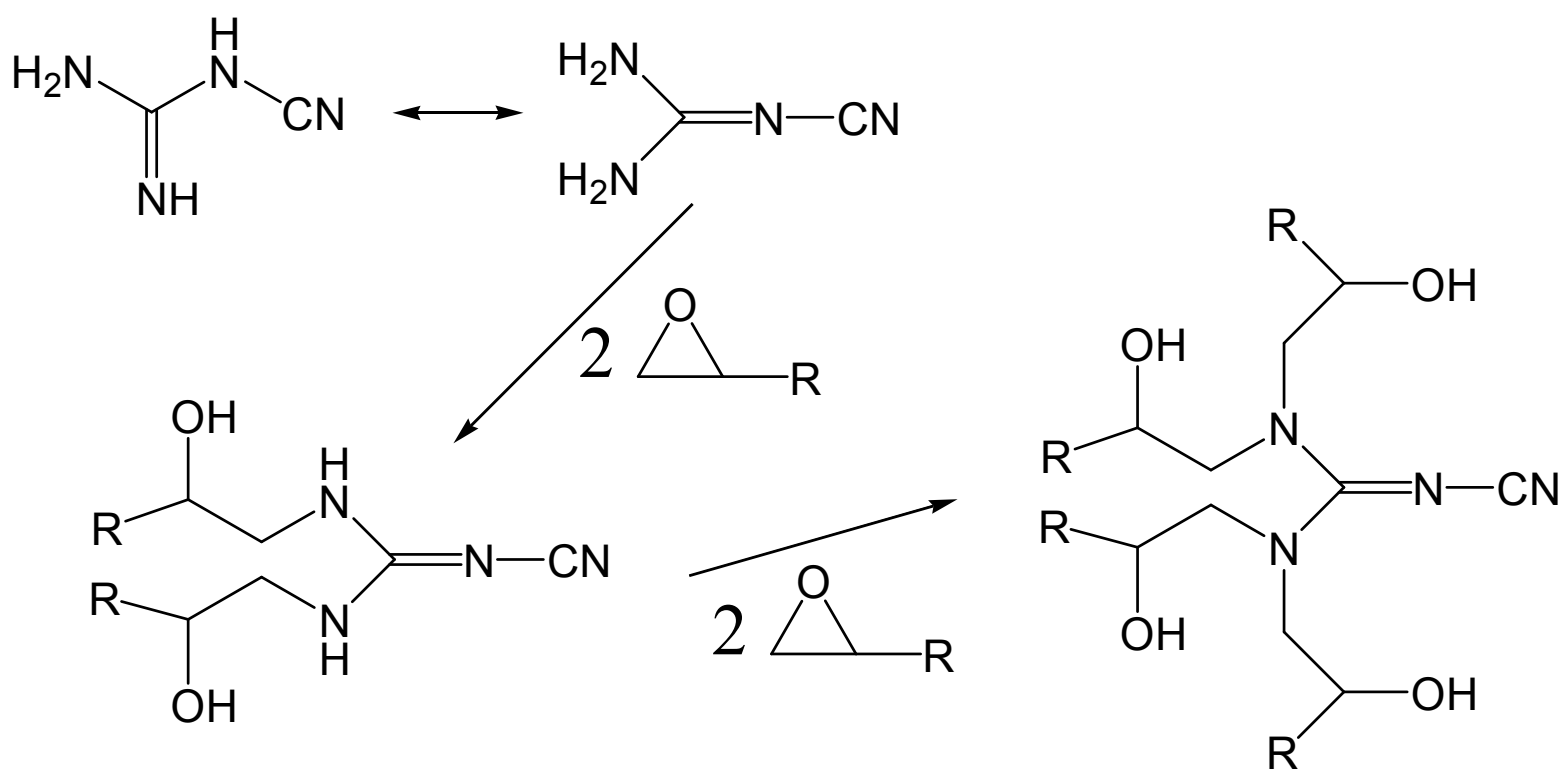

Figure S3. Curing mechanism between the epoxy and DICY. 


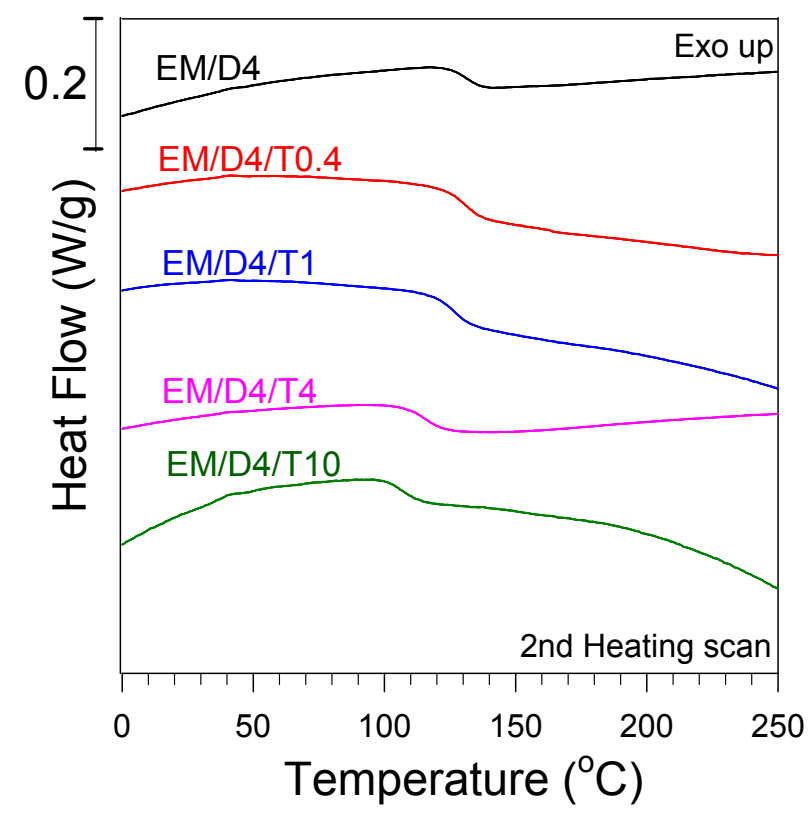

Figure S4. DSC results of epoxy/DICY/TDI-Uron with different TDI-Uron concentrations during second heating scan. 

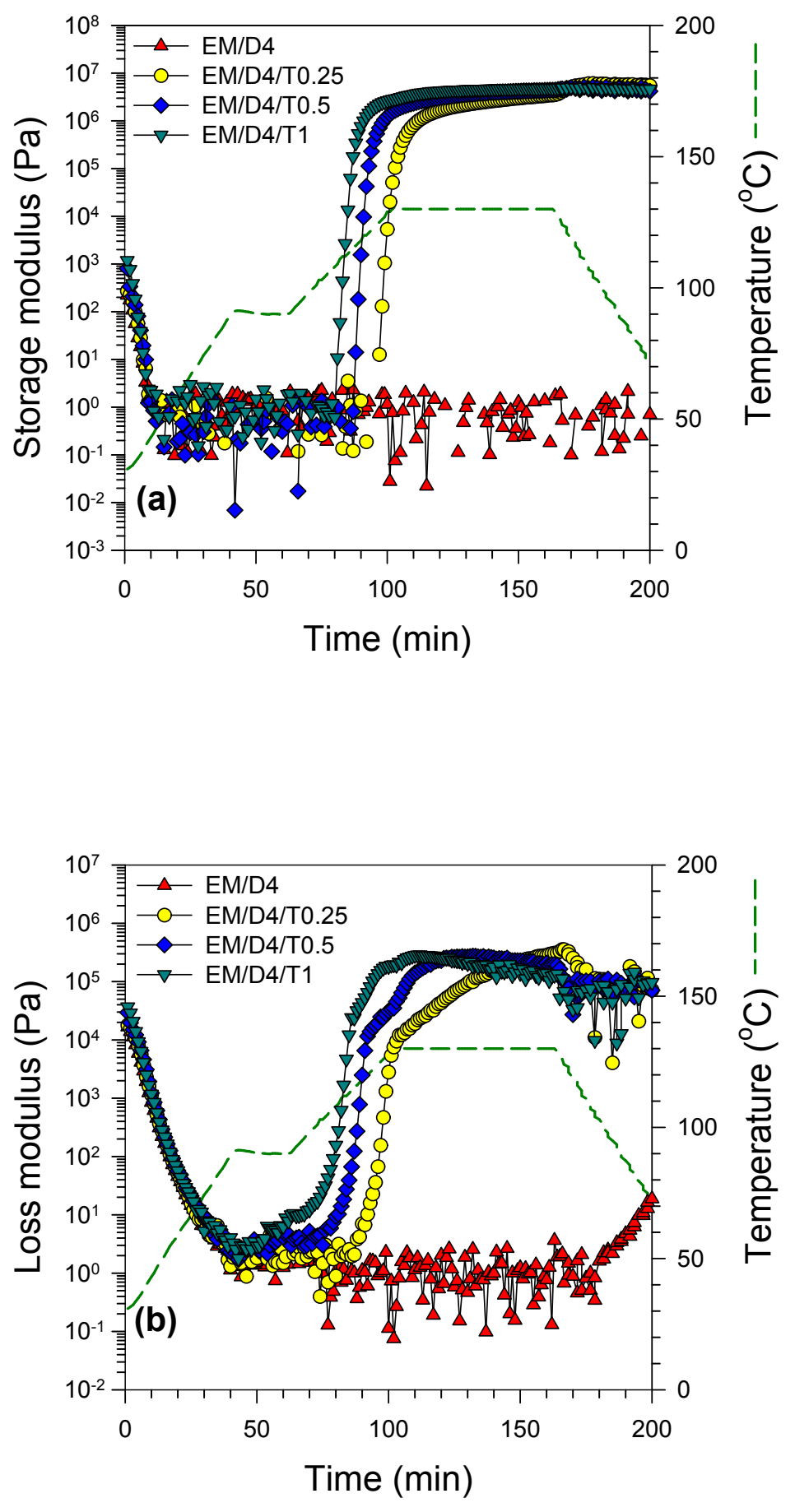

Figure S5. Storage (a) and loss (b) moduli of EM/D4/T with different TDI-Uron concentrations. 


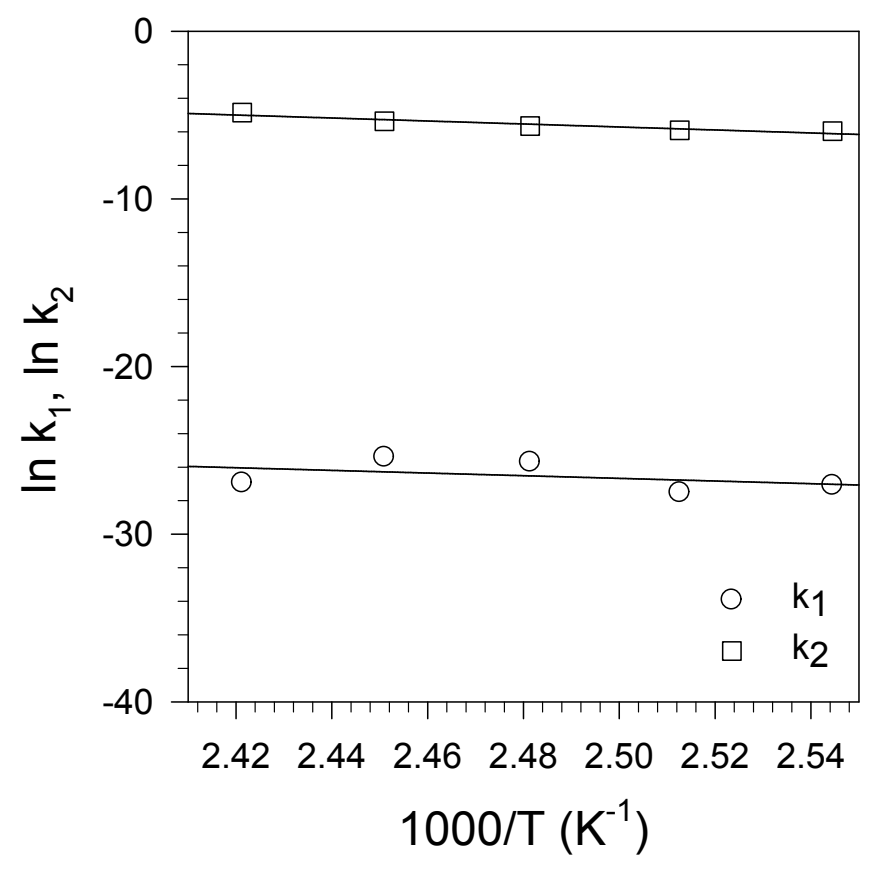

Figure S6. Arrhenius plot for finding activation energies and frequency factors from the rate constant vs. temperature data. 


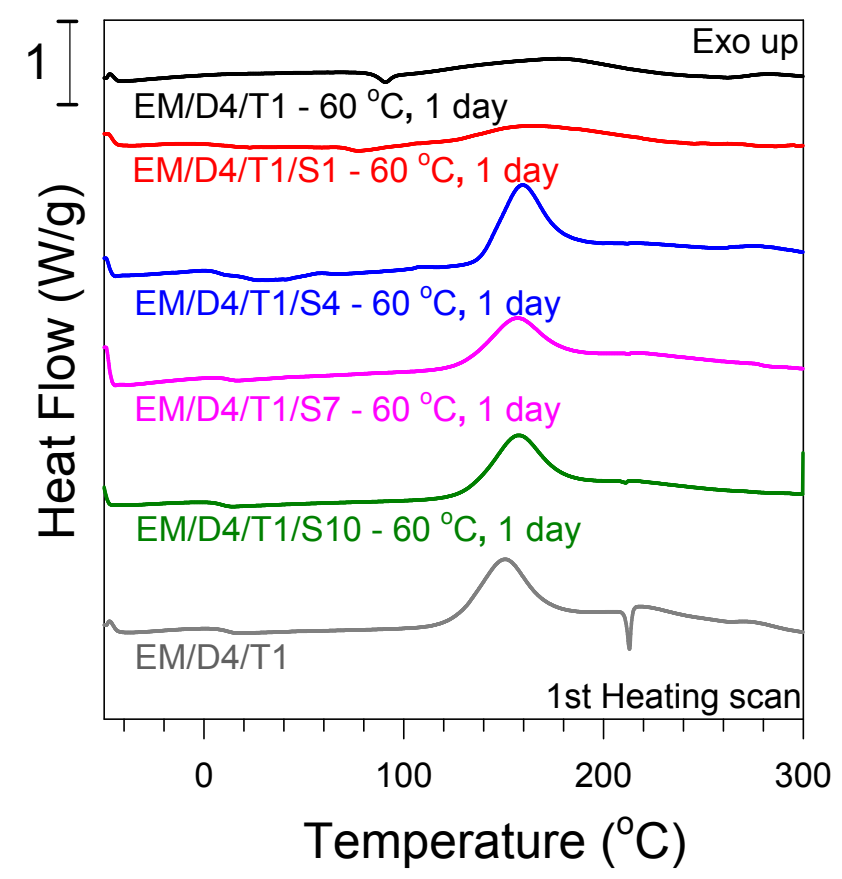

Figure S7. DSC results of EM/D4/T1 with different fumed silica concentrations. 


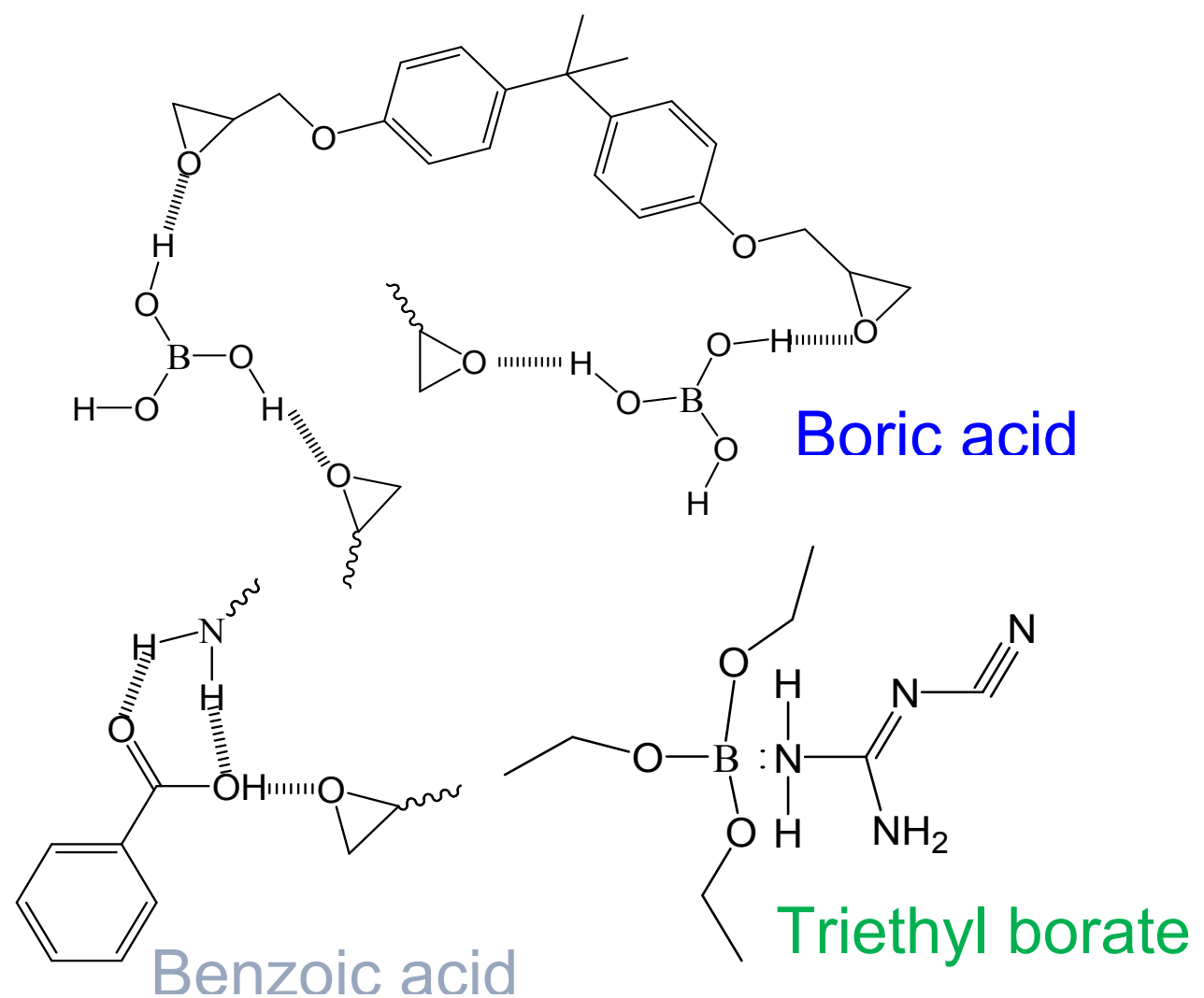

Figure S8. Proposed complex structures of epoxy resin containing BoA, BeA, and TEB. 

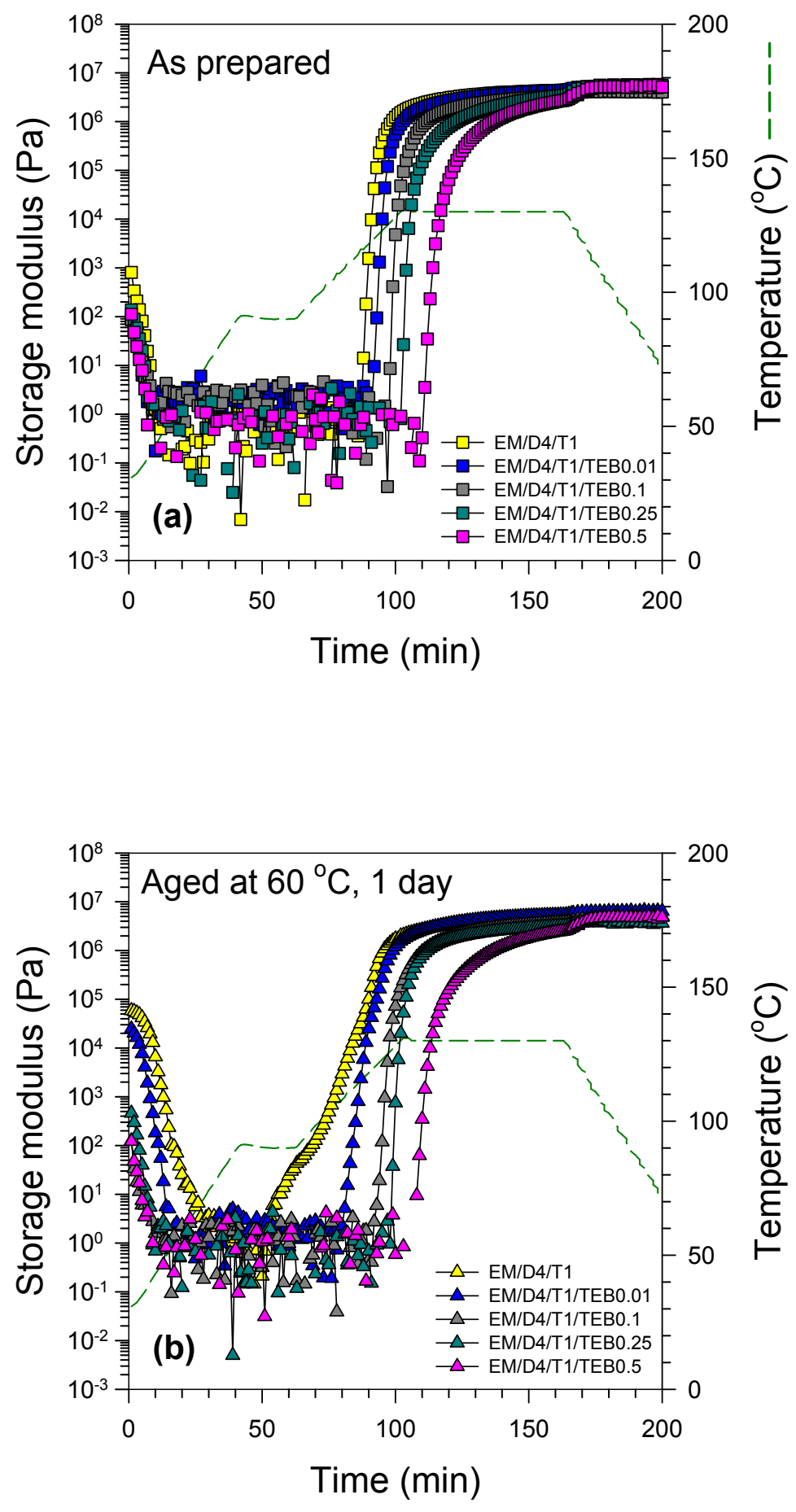

Figure S9. Storage modulus of EM/D4/T1/TEB resins at different TEB concentrations under two-step curing profile: (a) As-prepared samples and (b) Aged samples at $60{ }^{\circ} \mathrm{C}$ for 1 day. 

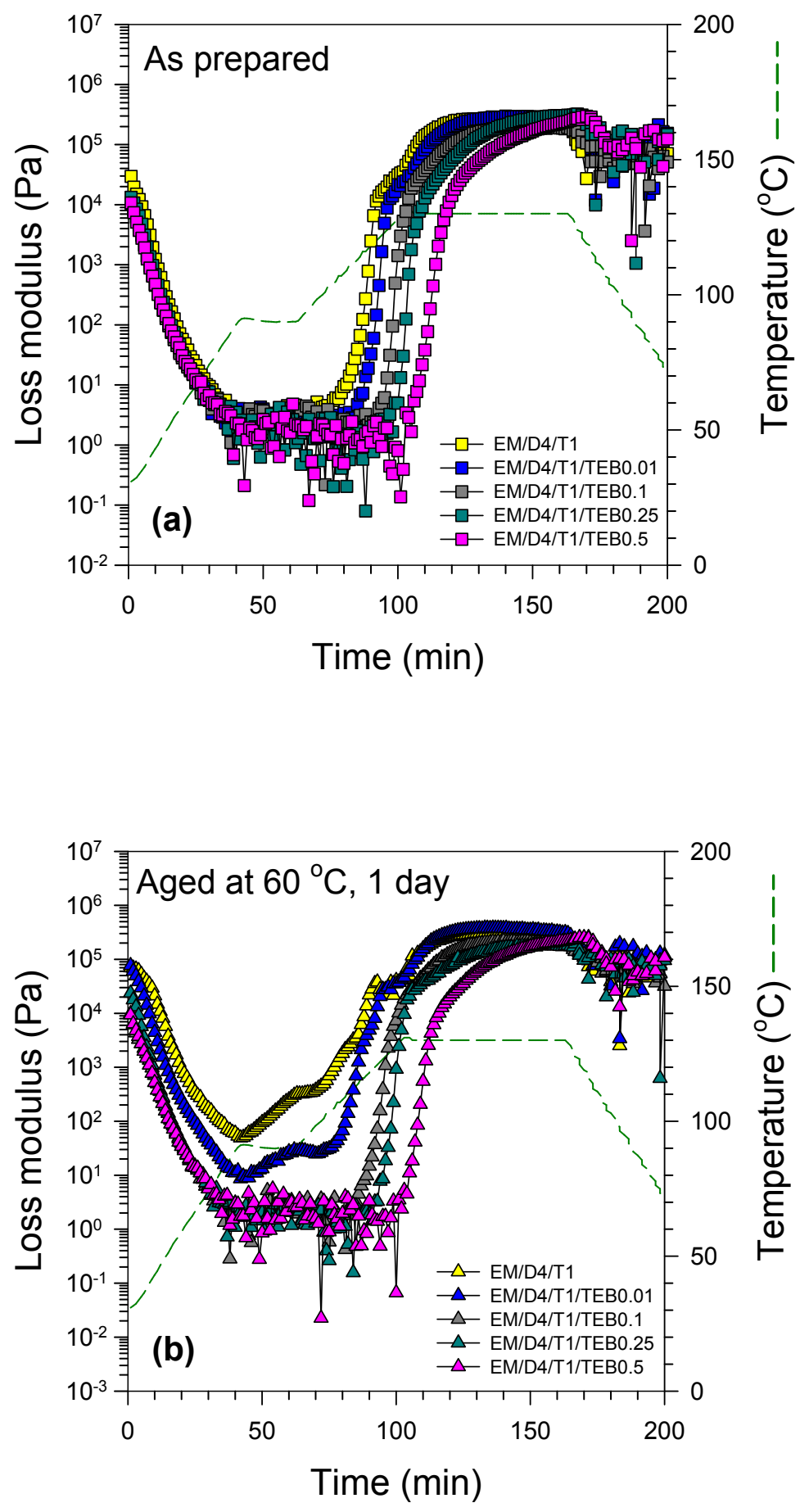

Figure S10. Loss modulus of EM/D4/T1/TEB resins at different TEB concentrations under two-step curing profile: (a) As-prepared samples and (b) Aged samples at $60^{\circ} \mathrm{C}$ for 1 day. 
Table S1. Samples for each measurement

\begin{tabular}{|c|c|}
\hline Measurement Equipment & Sample name \\
\hline Rheometer & $\begin{array}{c}\mathrm{EM} / \mathrm{D}(0,2,4,6, \text { and } 8), \mathrm{EM} / \mathrm{D} 4 / \mathrm{T}(0.2,0.4,1,2, \text { and } 4), \\
\mathrm{EM} / \mathrm{D} 4 / \mathrm{T}(0.25,0.5, \text { and } 1), \text { and } \mathrm{EM} / \mathrm{D} 4 / \mathrm{T} 1 / \mathrm{TEB}(0.01,0.1 \text {, } \\
0.25, \text { and } 0.5)\end{array}$ \\
\hline DSC & $\begin{array}{c}\mathrm{EM} / \mathrm{D}(2,3,4,6, \text { and } 15), \mathrm{EM} / \mathrm{D} 4 / \mathrm{T}(0.4,1,4, \text { and } 10), \\
\mathrm{EM} / \mathrm{D} 4 / \mathrm{T} 1 /(\mathrm{BoA} 1, \mathrm{BeA} 1, \text { and TEB} 1)\end{array}$ \\
\hline DMA & $\begin{array}{c}\text { Cured specimens of epoxy/CF prepreg } \\
\text { Epoxy resins used: EM/D4/T1/TEB0.1, HexPly M49, and } \\
\text { DT121H }\end{array}$ \\
\hline
\end{tabular}


Table S2. Summary of reaction order and rate constant from isothermal DSC data of EM/D4/T1 system by regression fitting

\begin{tabular}{ccccc}
\hline Temp. $\left({ }^{\circ} \mathrm{C}\right)$ & $m$ & $n$ & $k_{1}\left(\mathrm{~s}^{-1}\right)$ & $k_{2}\left(\mathrm{~s}^{-1}\right)$ \\
\hline 120 & 0.43 & 1.57 & $1.73 \mathrm{E}-12$ & $2.57 \mathrm{E}-03$ \\
130 & 0.33 & 1.67 & $6.90 \mathrm{E}-12$ & $3.49 \mathrm{E}-03$ \\
140 & 0.38 & 1.62 & $2.00 \mathrm{E}-12$ & $7.82 \mathrm{E}-03$ \\
\hline
\end{tabular}


Table S3. Summary of Arrhenius equation results for cure kinetics model-fitting

\begin{tabular}{ccc}
\hline & $k_{1}\left(\mathrm{~s}^{-1}\right)$ & $k_{2}\left(\mathrm{~s}^{-1}\right)$ \\
\hline Intercept & -6.85 & 16.59 \\
Slope & -7928 & -8918 \\
$k_{0}\left(\mathrm{~s}^{-1}\right)$ & $1.06 \mathrm{E}-03$ & $1.60 \mathrm{E}+07$ \\
$E_{a}(\mathrm{~J} / \mathrm{mol})$ & 65910 & 74140 \\
\hline
\end{tabular}


Table S4. Summary of DSC results of EM/D4/T1 with different fumed silica concentrations

\begin{tabular}{|c|c|c|c|c|}
\hline Sample & $\Delta H(\mathrm{~J} / \mathrm{g})$ & $T_{\text {peak }}\left({ }^{\circ} \mathrm{C}\right)$ & $T_{g}\left({ }^{\circ} \mathrm{C}\right)$ & $\begin{array}{l}T_{g} \text { of cured } \\
\text { sample }\left({ }^{\circ} \mathrm{C}\right)\end{array}$ \\
\hline EM/D4/T1 & 220 & 148 & 10 & 124 \\
\hline $\begin{array}{c}\mathrm{EM} / \mathrm{D} 4 / \mathrm{T} 1 \\
60{ }^{\circ} \mathrm{C}-1 \text { day }\end{array}$ & 90 & 175 & 85 & 133 \\
\hline $\begin{array}{l}\mathrm{EM} / \mathrm{D} 4 / \mathrm{T} 1 / \mathrm{S} 1 \\
60^{\circ} \mathrm{C}-1 \text { day }\end{array}$ & 120 & 159 & 70 & 131 \\
\hline $\begin{array}{l}\mathrm{EM} / \mathrm{D} 4 / \mathrm{T} 1 / \mathrm{S} 4 \\
60^{\circ} \mathrm{C}-1 \text { day }\end{array}$ & 170 & 158 & 20 & 129 \\
\hline $\begin{array}{l}\mathrm{EM} / \mathrm{D} 4 / \mathrm{T} 1 / \mathrm{S} 7 \\
60^{\circ} \mathrm{C}-1 \text { day }\end{array}$ & 190 & 157 & 13 & 126 \\
\hline $\begin{array}{c}\mathrm{EM} / \mathrm{D} 4 / \mathrm{T} 1 / \mathrm{S} 10 \\
60^{\circ} \mathrm{C}-1 \text { day }\end{array}$ & 210 & 154 & 10 & 119 \\
\hline
\end{tabular}

